\title{
A 25 YEARS OLD MALE WITH PRIMARY DUODENAL LYMPHOMA
}

\author{
AHMEDUL KABIR ${ }^{1}$, FAIZUL ISLAM CHOWDHURY², JAYANTA BANIK ${ }^{3}$, UMME KULSUM MITU ${ }^{4}$
}

\begin{abstract}
Primary duodenal lymphoma is very atypical, although the gastrointestinal (GI) tract is the most prominent site of extra nodal lymphoma. Here we present a case of 25 years old male, presented with upper abdominal pain and vomiting and eventually diagnosed as a case of duodenal non Hodgkin's lymphoma. The particular interest in this report is the fact that, it is important to have a high index of suspicion to diagnose primary intestinal lymphoma as it has high mortality which requires early diagnosis and treatment.
\end{abstract}

\section{Introduction}

Lymphoma is a heterogeneous disease as there is a great variety of biology of normal lymphocytes and clinical behavior is different. The World Health Organization (WHO) classification divides lymphoma biologically into B- Cell and T- Cell / Natural Killer (NK) cell lineages which are commonly called non Hodgkin's lymphoma. The incidence of the various subtypes varies widely as some lesions appear to be peculiar to particular sites and ethnic groups. ${ }^{1}$ Primary non-Hodgkin's lymphoma of the duodenum is an uncommon primary tumor of the gastrointestinal (GI) tract which accounts for less than $12 \%$ of all nonHodgkin's lymphoma (NHL). ${ }^{2,3}$ The majority of these lymphomas arise in the stomach, with less than $30 \%$ arising in the small intestine. ${ }^{3}$ The incidence of lymphoma decreases from the ileum to jejunum to duodenum. ${ }^{1}$ Alexander reported the first case of duodenal lymphoma in $1877 .{ }^{4}$ Among extranodal NHL, Diffuse, large B- cell lymphoma is the most common histologic type of primary extranodal lymphoma that arises in the gastrointestinal tract. ${ }^{2}$

But in the early 1980s mucosa associated lymphoid tissue (MALT)-derived lymphoma (MALT lymphoma) was recognized as a distinct clinicopathological entity. Gastric MALT lymphoma is associated with Helicobacter pylori (H. pylori) infection, and the eradication of $\mathrm{H}$. pylori results tumor regression. However, MALT lymphoma of the duodenum is very rare, and may be resistant to $\mathrm{H}$. pylori eradication. ${ }^{3}$ Among other types of lymphoma Mantle cell lymphoma, and mature T-cell lymphoma can also occur. Lymphoma of the small bowel usually present with local gastrointestinal symptoms, which includes malabsorption, mass lesions, bowel obstruction, bleeding, and/or systemic symptoms like fever, weight loss and night sweats. ${ }^{1}$ We are reporting a patient who presented to our institution with acute abdomen and diagnosed as primary Non Hodgkin's Lymphoma (NHL) of the gastrointestinal tract.

\section{Case report}

A 25 years old, previously healthy male, presented to us with upper abdominal pain and vomiting for two months. The pain was located in the epigastric region, colicky in nature and radiating towards the back associated with vomiting of ingested food material. There were no systemic symptoms and his bowel and bladder habit were normal.

On examination he was hemodynamically stable with mild anaemia and no lymphadenopathy. Per abdominal examination reveals slightly distended abdomen with rigid abdominal wall. There was mild tenderness in the epigastric region and bowel sound was sluggish. Other systemic examination were normal.

On investigation, Complete blood count and Blood film revels, WBC - $14.5 \mathrm{X} 10 \times 9 / \mathrm{L}$ with a PMN predominance of $74 \%$, Hematocrit- $39.28 \%$, Platelets - 250 X 10x9 /L, ESR - 20 mm/1st Hr, Hb ( Cyn. Meth. ) $-13.5 \mathrm{~g} / \mathrm{dl}$ (normal is $13-17 \mathrm{~g} / \mathrm{dl}$ ), PBF : RBC - Normocytic and normochromic, WBC - Mature with Neutrophil Leukocytosis, Plateletes - Normal. Urinalysis was negative for protein or red cells. Blood

1. Assistant Professor, Department of Medicine, Dhaka Medical College, Dhaka

2. Associate professor, Department of Medicine, Dhaka Medical College, Dhaka

3. Post-graduate Trainee, Dept. of Medicine, Bangabandhu Sheikh Mujib Medical University, Dhaka

4. Post-graduate Trainee, Dept. of Medicine, Dhaka Medical College Hospital, Dhaka

Correspondence : Dr. Ahmedul Kabir, Assistant Professor, Department of Medicine, Dhaka Medical College, Dhaka 


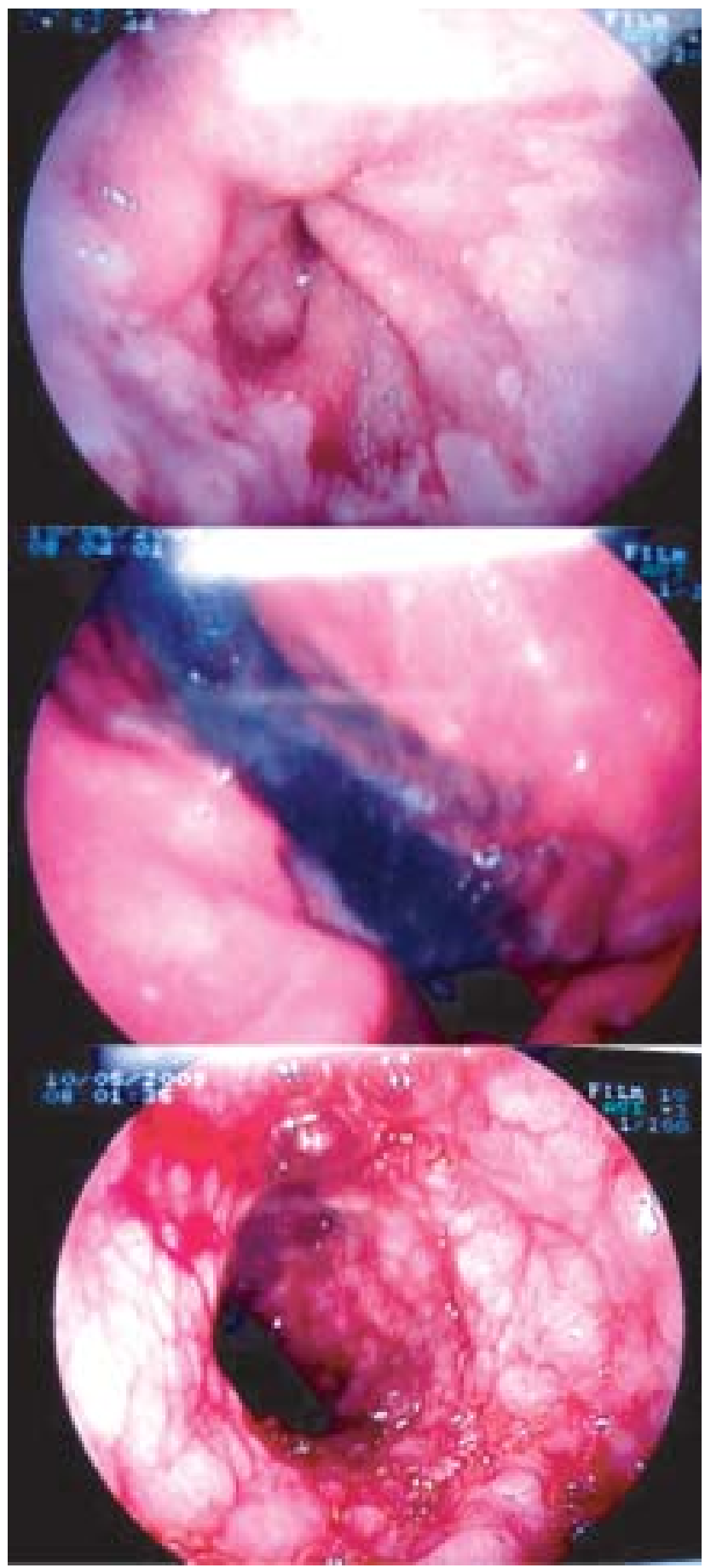

Fig.-1: Upper GIT endoscopy of this patient showing multiple nodular lesion in the second part of duodenum.

urea, electrolytes and S. createnin was normal. LFT shows, S.Bilirubin - $1.2 \mathrm{mg} / \mathrm{dl}$, ALT(SGPT) - $20 \mathrm{U} /$ L, AST - 18 U/L, Alkaline phosphatase - $45 \mathrm{U} / \mathrm{L}$, Prothrombin time - $11.5 \mathrm{sec}$ ( control $12 \mathrm{sec}$ ). Chest radiograph is normal. USG of whole abdomen shows multiple hypoechoic focal thickening in different parts of small intestine suggesting lymphomatus involvement. Endoscopy of upper GIT shows multiple nodular lesion in the second part of duodenum and scope could not be negotiated further due to narrowing of the lumen (Fig.-1). Biopsy from the nodule was taken and send for histopathology. The histopathologic sections show intestinal tissue infiltrated by immature atypical lymphoreticular cells. Some of these show plasmacytoid appearance with relatively large nucleus having loose chromatin. Most of the cells are monotonus in appearance. The tumor cells have been infiltrated in the muscle layer. Features are compatible with Non Hodgkin's lymphoma, lymphoplasmacytoid type, intermediate grade.

\section{Discussion}

Lymphomas are malignant neoplasms characterized by the proliferation of cells native to the lymphoid tissues, that is, lymphocytes, histocytes, and their precursors and derivatives. They are broadly classified into Hodgkin's (HL) and Non Hodgkin's lymphoma (NHL). ${ }^{4}$

Primary extranodal lymphomas are a heterogeneous group of diseases which have diverse etiology, pathogenesis, pattern of presentation and outcome. The commonest site of extra nodal NHL is the GI tract, but virtually every extranodal location has been reported. ${ }^{5}$ Of these $50-60 \%$ occur in the stomach and $20-30 \%$ in the small intestine. In the small intestine, the commonest site is the ileocaecal region and the duodenum is the least common. In the GI tract the incidence of lymphoma depends on the amount of lymphoid tissue present in a particular segment. ${ }^{4}$

Among these, B-cell type, diffuse large-cell type and lymphoplasmacytoid histology are common; follicular histology, Burkitt's lymphoma and Mental cell lymphoma (MCL) are less common. Mucosa-associated lymphoid tissue lymphoma that are usually localized and have an excellent prognosis are leveled as lowgrade which include gastric, salivary gland, Waldeyer's ring and thyroid, orbital and low-grade lymphomas of the breast. The intermediate-grade lesions of diffuse large-cell and B-lineage occur commonly in Waldeyer's ring, paranasal sinus, thyroid, breast, intestine, bone which are also highly curable with combined modality therapy. ${ }^{5}$

Most of the patients with gastrointestinal lymphoma present with vomiting, abdominal pain, weight loss, and fever. ${ }^{5}$ Other features like early satiety, symptoms 
of peptic ulceration, bleeding in the form of haematemesis, malaena or anaemia or non-specific symptoms like loss of appetite may also present. Bleeding DU is rare as a presentation of duodenal lymphoma. ${ }^{6}$ Few cases of primary gastrointestinal lymphoma, particularly primary duodenal lymphoma can also presents as jaundice. ${ }^{4}$

Lymphoplasmacytoid lymphoma or other small B-cell lymphoproliferative disorders are generally presented as Waldenström macroglobulinemia (WM) and its associated hyperviscosity syndrome (HVS). In patients with clinicopathologic features of WM, MALT-type (extranodal marginal zone B-cell-mucosa-associated lymphoid tissue lymphoma ) should also be considered in the differential diagnosis. ${ }^{7}$

Follicular lymphoma (FL) is one of the most frequent B-cell lymphomas which are more frequently found in the duodenum than in other areas of the gastrointestinal tract. They often present with multiple polyps around the ampulla of Vater and are usually localised tumours. ${ }^{8}$

The following criteria need to be met, in order to diagnose the primary GI tract lymphoma,

1) absence of palpable superficial lymphadenopathy;

2) absence of enlargement of mediastinal lymph nodes on chest x-ray;

3) normal leukocyte and differential counts;

4) predominance of alimentary tract lesions with only regional lymph node involvement; and

5) absence of tumor involvement of the liver and spleen. ${ }^{5}$

Our case fulfilled all of the above criteria .

For histologic diagnosis an adequate wedge-type (not FNA) biopsy is necessary. Proper handling of the specimen is needed and fresh tissue is to be send to the pathology laboratory for proper immuno-staining and molecular lineage clarification. It is necessary for classification, and appropriate treatment. because specific treatment is needed for different lineage and various histologic subtypes of lymphoma. ${ }^{1}$ Upper GIT Gastroduodenoscopy is the effective modality of investigation for taking the biopsy.

According to literature, chemotherapy is suggested as the most successful treatment for primary GI tract lymphoma cases. ${ }^{4}$ Systemic chemotherapy with cyclophosphamide, vincristine, and prednisolone (CVP) showed complete remission of a duodenal MALT lymphoma. Monotherapy with cyclophosphamide or clarithromycin may be a reasonable option for MALT lymphoma confined to the mucosa or submucosa of the duodenum. ${ }^{1}$ R-CHOP (rituximabcyclophosphamide, doxorubicin, vincristine and prednisone) chemotherapy is used successfully in duodenal diffuse large B cell lymphoma. ${ }^{4}$ Chemotherapy, monoclonal antibody based therapies and autologous and allogenic stem cell transplantation are current modalities of treatment available for mantle cell lymphoma. ${ }^{5}$ Duodenal follicular lymphoma showed successful remission in radiation therapy. 6 Surgery is another good option for duodenal non Hodgkin's lymphoma which include pancreaticoduodenectomy followed by postoperative radiotherapy, and total resection of all bulky disease followed by chemotherapy without radiotherapy. Although H. pylori eradication therapy showed regression in gastric MALT lymphoma, for duodenal MALT lymphoma, H. pylori eradication did not lead to lymphoma regression and in contrast to gastric MALT lymphoma $H$. pylori eradication did not cure duodenal MALT lymphoma. ${ }^{2}$

Primary GI lymphoma is an aggressive malignancy, with an overall 5-year survival rates is of $47 \%, 5$-year disease-free survival is $40 \%$, and $79 \%$ of mortality occurring within the first year of diagnosis. Important prognostic factors include stage of the disease, extent of surgical resection, response to treatment, serosal involvement, multimodality treatment, and performance status of the patient. ${ }^{8}$ GI lymphoma can result in death even with therapy. So the best outcome for the patient depends on the early diagnosis and treatment of the disease. ${ }^{9}$

\section{Conclusion}

In conclusion, especially in younger population, with unexplained upper abdominal pain, a diagnosis of lymphoma should be considered. Upper GIT endoscopy is a good modality of investigation to diagnose any lesion in the stomach and duodenum. Though most DUs are benign peptic ulcers, the possibility of other pathologies such as lymphoma should be considered and edge biopsies should be done especially if the ulcer is atypical in appearance or location. Timely diagnosis of the patient and commencement of the appropriate treatment is necessary as earlier stage at the time of presentation influences the outcome of these patients. Therefore, it is important to have a high index of suspicion to diagnose primary duodenal lymphoma as it is a potentially curable disease. The future approach to management of extranodal lymphomas 
may have more basis in the etiology and pathogenesis of these disorders than purely on histological appearance and anatomic extent. Better understanding of the immune response and its relation to lymphoproliferative disorders may ultimately lead to a lesser focus on ablative cytotoxic therapies and an increased emphasis on specific etiological determinants and the control of aberrant immune response.

\section{References}

1. Hansen PB, Vogt KC, Skov RL, et al. Primary gastrointestinal non-Hodgkin's lymphoma in adults:a population-based clinical and histopathologic study. Journal of Internal Medicine 1998; 244: 71-78.

2. Muchmore JH, Haddad CG, Goldwag S., Primary non-Hodgkin's lymphoma of the duodenum. Am Surg 1994 ;60: 924-8.

3. Woo KH, Kim JH, Yoon SB, et al. Duodenal MucosaAssociated Lymphoid Tissue Lymphoma: A Case Report. The Korean Journal of Internal Medicine 2007; 22: 296-299.
4. Rahman S, Reyes E, Mehta A, Virk Z, Gintautas J, Cervantes J . Primary Duodenal Lymphoma presenting as Obstructive Jaundice. The Internet Journal of Gastroenterology. 2009;7(2).

5. Mary K. Gospodarowicz, Sutcliffe SB. The extranodal lymphomas, Seminars in Radiation Oncology 1995;5:281-300.

6. Mundasad B , Hawe M. Lymphoma Presenting As A Bleeding Duodenal Ulcer: A Case Report. The Internet Journal of Tropical Medicine. 2006;3(1).

7. Riccardo Valdez, William G. Finn, et al. Waldenström Macroglobulinemia Caused by Extranodal Marginal Zone B-cell Lymphoma: A Report of Six Cases. Am J Clin Pathol 2001;116:683690.

8. Sato Y, Ichimura K, Tanaka T,et al. Duodenal follicular lymphomas share common characteristics with mucosa-associated lymphoid tissue lymphomas. Journal of Clinical Pathology 2008;61:377-381. 